\title{
Ton du discours intérieur dans les romans de Stendhal
}

\author{
Laure Lassagne
}

\section{OpenEdition}

1 Journals

Édition électronique

URL : http://journals.openedition.org/recherchestravaux/356

DOI : 10.4000/recherchestravaux.356

ISSN : 1969-6434

\section{Éditeur}

UGA Éditions/Université Grenoble Alpes

\section{Édition imprimée}

Date de publication : 15 juillet 2009

Pagination : 109-120

ISBN : 978-2-84310-146-5

ISSN : 0151-1874

\section{Référence électronique}

Laure Lassagne, "Ton du discours intérieur dans les romans de Stendhal », Recherches \& Travaux [En ligne], 74 | 2009, mis en ligne le 28 février 2011, consulté le 07 septembre 2020. URL : http:// journals.openedition.org/recherchestravaux/356; DOI : https://doi.org/10.4000/recherchestravaux. 356 
Laure LASSAGNE

Université Paris 4

\section{Ton du discours intérieur dans les romans de Stendhal}

\section{Introduction}

La plupart des analyses littéraires et linguistiques associent le ton à l'investissement de l'énonciateur dans son discours. Il est un indice de la présence, du positionnement du locuteur, du rapport qu'il noue avec son allocutaire. À en croire Paul Valéry, Stendhal en apporterait une illustration exemplaire : le ton des écrits d'Henri Beyle «dénonce ${ }^{\mathrm{I}} »$ immédiatement leur auteur, dans quelque page que ce soit. Son ton est «le plus individuel qu'il soit en littérature», un «ton si marqué [qui] fait l'homme si présent» (ibid.,p. 210).

Cette approche du ton a conduit certains critiques à étudier les effets de voix dans la narration stendhalienne et à examiner le type de rapports qu'établit le locuteur avec son allocutaire au sein de la fiction, dans les dialogues romanesques dont Stendhal spécifie très souvent le ton², ou au-delà : Gilles Philippe décrit ainsi le ton de Stendhal par l'ambivalence du lien que l'auteur contracte avec son lecteur auquel tout à la fois son texte s'adresse et dont il affirme être détaché.

L'appréhension du ton se complique lorsqu'on aborde les nombreux monologues qui contribuent fortement à donner aux romans stendhaliens leur ton si caractéristique. Aucun de ces deux modes d'approche ne fonctionne

I. «Ce qui frappe le plus dans une page de Stendhal, ce qui sur-le-champ le dénonce, attache ou irrite l'esprit, - c'est le Ton» (P. Valéry, «Stendhal», dans Variété I et II [1924-1930], Gallimard, «Folio Essais», 2002, p. 209).

2. On relève 98 occurrences du terme «ton» dans Lucien Leunven. Stendhal prend soin de caractériser le «ton» des dialogues romanesques. Une seule occurrence s'applique à un monologue intérieur. 
parfaitement. Le ton des discours intérieurs ne peut être envisagé sous l'angle dialogique, pour une raison évidente : les monologues, cette parole gardée pour soi, échappe à toute situation d'échange au niveau intrafictionnel. Toute caractérisation des rapports entre interlocuteurs est vaine et déplacée.

D'autre part, il semble difficile d'assimiler les monologues stendhaliens à une voix intérieure. La comparaison du monologue littéraire à une voix silencieuse appartient à un paradigme postérieur, de la fin du XIX ${ }^{\mathrm{e}}$ siècle et du début du $\mathrm{Xx}^{\mathrm{e}}$ siècle, paradigme dont l'application aux productions des années I 830 est pour le moins risquée. L'attention portée par Stendhal à l'énonciation, souvent dramatisée, dans des monologues ponctués d'italiques, d'exclamations, d'interrogations, n'implique pas pour autant la notion de voix. Certes, la «petite vanité», «le parti de la tentation», «le parti de la prudence» ou le «parti de l'amour ${ }^{3} »$ «se parlent» voire «s'écrient» dans les discours intérieurs stendhaliens, mais toutes ces formules doivent être entendues comme autant de métaphores dans une sorte de théâtralisation du débat intérieur. La voix désigne ici par métaphore un point de vue. Le dialogue et les répliques entre les différents partis doivent être lus dans un sens intellectuel et logique. Cette mise en scène s'inscrit dans une tradition rhétorique où les délibérations prennent la forme d'une disputatio, sans qu'il soit question de prononciation à voix haute.

Les monologues stendhaliens engagent donc à envisager le ton en dehors de toute perspective vocale. Ils contraignent à appréhender autrement cette notion fuyante, ses composantes et ses fondements textuels. Sans doute faut-il aborder le ton comme une catégorie pragmatique. De fait, les toutes premières études du monologue stendhalien ont souligné le ton injonctif de soliloques en prise directe avec l'action des personnages. Dominique Trouiller commence son article de 1969 sur les monologues du Ronge et le Noir en notant que "[1]e ton constant de ces monologues est celui de l'ordre que l'on se donne à soimême; le «il faut» est son verbe; le futur son mode : ce sont ses marques les plus caractéristiques ${ }^{4} \gg$.

Les personnages stendhaliens multiplient les ordres dans leurs soliloques et s'enjoignent à suivre une ligne de conduite. Pourtant, ces admonestations ne représentent que la partie émergée de l'iceberg. Car le ton passe essentiellement pour Stendhal par l'effacement de l'énonciateur, du message et des volontés dont il charge son discours. De même qu'Henri Beyle expliquait à sa sœur que le ton en société, le bon ton, cherche à faire effet mais ne s'entend qu'«à demi-mot'», de même dans ses textes, le ton doit savoir se faire discret

3. Voir par exemple Lucien Leunen, p. 336-337.

4. D. Trouiller, «Le monologue intérieur dans Le Rouge et le Noir», Stendhal Club, n 43 , is avril i 969 , p. 246.

5. Stendhal reproche précisément aux auteurs contemporains de «charger» excessivement 
et s'entendre entre les phrases. Aussi, nous avancerons l'hypothèse que le ton, la visée pragmatique des monologues, se marque essentiellement par le rythme. Si l'on suit Stendhal qui envisageait le rythme comme "la liaison des idées ${ }^{6} »$, le ton s'inscrirait pour l'essentiel dans le mode d'enchaînement des propositions. Dans la conception indicielle que Stendhal a du langage - langage qui traduit mais ne peut exprimer directement les sentiments -, le rythme semble faire exception. En effet, pour peu que l'auteur adopte un style naturel, le rythme permet d'établir selon Beyle «un accord entre les pensées et les tournures ${ }^{7}$. En conséquence, il est apte à «montrer les sentiments ${ }^{8} »$ de l'énonciateur. La progression, la construction de la phrase est capable de montrer le mode d'engendrement des pensées et, plus largement, les mouvements intimes.

Le ton procéderait donc de la mise en tension de deux segments langagiers. L'étymologie du terme conforte cette hypothèse. «Ton» vient du grec tonos, dont le sens est physiologique : ce sont les tendons, les ligaments tendus et, dans un sens abstrait, tonos désigne l'action de tendre. Le XVIII ${ }^{\mathrm{e}}$ siècle réactualise ce sens étymologique et le Grand Dictionnaire universel du XIX siècle de Pierre Larousse l'entérine : le ton est l'état de tension, d'élasticité des tissus organiques et, dans un sens moral, la vigueur, l'énergie".

Si l'on examine la tension du tissu textuel, la volonté et le désir de l'énonciateur sont particulièrement perceptibles dans les articulations du discours, qui fondent le ton. Nous essaierons de comprendre comment en parcourant tous les degrés d'élasticité : les cas d'hypertension, lorsque les segments langagiers sont reliés les uns aux autres par un lien qui semble être de quasi-nécessité, avant d'examiner les cas plus problématiques où l'impression est celle d'un ton détaché, où les liens semblent se détendre jusqu’à devenir comme flottants. Tout notre effort visera à dépasser le constat de simple mimétisme, du rythme d'une phrase imitant son sens ${ }^{10}$. Nous nous efforcerons de suivre

le ton, et de le fausser ce faisant: «Pour le ton du jour je le vois chargé en bien ou en mal chez tous les auteurs, et peint tel qu'il [n'] est nulle part» (Correspondance, t. I, p. 252-253. Lettre à Pauline Beyle de novembre i 805 ).

6. «Le rythme se fonde presque entièrement sur la liaison des idées.» Quant à la liaison des idées, elle ne vient «que de la liaison des sensations» (Journal littéraire, t. II, p. 85-86).

7. Journal littéraire, t. II, p. 85 . Nous soulignons.

8. «En fait de style, bien réellement, et sans phrase de journal, la forme fait partie de la chose. Une transposition de mots montre l'objet d'un autre côté. Pour les sentiments, le rythme les montre» (ibid., p. 80).

9. P. Larousse dir., Grand Dictionnaire universel $d u$ XIX siècle [1876], Slatkine, i982, t. XV, p. 285 . Notons que ce sens est loin d'être marginal. Il figure en bonne place, avant la définition comme «caractère propre aux manières» du «bon ton» et du «mauvais ton».

Io. Constats de mimétisme auxquels nous sommes souvent conduits, lorsque nous avançons que le rythme précipité d'une phrase reflète l'émotion du locuteur ou qu'à l'inverse le rythme languissant d'un paragraphe traduit sa profonde lassitude. 
plutôt la voie ouverte par Henri Meschonnic lorsqu'il avance que «le rythme n'est pas le sens, ni redondance, ni substitut, mais matière de sens, même la matière du sens ${ }^{1 \mathrm{II}} \gg$.

\section{Ton dramatique et hypertension logico-syntaxique}

D’une manière générale, le récit stendhalien se montre parcimonieux en mots de liaison et préfère la juxtaposition ou la parataxe. Jean Prévost constate ainsi que notre auteur «se sert peu des articulations logiques du discours» : «il tient du XVIII ${ }^{\mathrm{e}}$ siècle l'habitude de les remplacer par une simple juxtaposition des idées, dans leur ordre le plus parfait ${ }^{12} \gg$. Aux yeux de Jean Prévost, l'ellipse des particules de liaison permet de donner au récit le tempo de la pensée, elle lui confère cet «allegro intime, ce staccato grêle et un peu $\sec ^{13}$ » dans lesquels Julien Gracq voyait le génie de Stendhal.

Pourtant, contre toute attente, la figuration romanesque du discours intérieur présente une concentration remarquable de mots de liaison. Dans leurs monologues, les héros stendhaliens explicitent volontiers les enchaînements logiques. Au-delà même des longs monologues délibératifs où l'explicitation du raisonnement est nécessaire pour résoudre un éventuel dilemme, tous les monologues multiplient les connecteurs logiques : mots coordonnants (mais, car, donc, et...), adverbes (d'ailleurs...), interjections (eh bien ...), conjonctions de subordination (puisque, si...). Ce sont souvent ces mots de liaison qui révèlent le mieux les dispositions du locuteur et donnent le ton. Ainsi que le remarquait Éric Bordas, "[] e discours et son sujet sont dans le liant, dans l'inscription d'une logique, temporelle ou cognitive ${ }^{14} \gg$. L'inquiétude et les désirs du personnage se laissent percevoir dans les articulations logico-syntaxiques

I I. H. Meschonnic, Critique du rythme. Anthropologie historique du langage, Lagrasse, Verdier, I9 82, p. 83

I 2. J. Prévost, La Création chez, Stendhal [1951], Gallimard, «Folio Essais», I996. J. Attuel va dans le même sens : «Stendhal supprime volontiers les particules de liaison» (Le Style de Stendhal, Bologne-Paris, Patròn-Nizet, I 980, p. 266).

I3. Dans En lisant, en écrivant, J. Gracq tente de cerner la nature du génie de Stendhal : «Il n'a ni grande invention, et il le sait (il lui faut la béquille du fait divers) ni grande technique (quoiqu'il s'en vante) ni grande imagination (et il s'en moque) ni, autant qu'on le dit, de cette "profondeur psychologique" qui est surtout chez lui vivacité de la formule et ingéniosité du trait - rien que cet allegro intime, ce staccato grêle et un peu sec qui n'est qu'à lui, mais au rythme duquel la vie se remet [...] irrésistiblement à danser» (En lisant, en écrivant, José Corti, I98 I, p. 46).

I4. É. Bordas, «Et la conjonction resta tensive. Sur le et de relance rythmique» (Le Français moderne, $\mathrm{n}^{\circ} \mathrm{I}, 2005$, p. 23). 
mieux que dans nombre de substantifs ou de verbes de sentiments passant pour expressifs.

Prenons par exemple l'un des outils grammaticaux fort appréciés des personnages s'employant à clarifier leur situation affective et relationnelle : la conjonction de subordination de condition si. Dans la configuration la plus fréquente, parmi la cinquantaine d'occurrences relevées au sein des monologues de La Chartreuse de Parme, si est suivi d'un verbe conjugué au présent de l'indicatif et la principale est elle aussi au présent de l'indicatif. Le personnage est tendu vers ce qui va arriver et il en imagine les répercussions immédiates. Le ton haletant résulte de la tension d'un personnage sur le qui-vive.

Si la duchesse part, je la suis, se disait [le comte Mosca]. (La Chartreuse de Parme, p. 253)

La subordonnée introduite par si est quasiment toujours placée en tête : la syntaxe épouse le mouvement de projection du personnage qui se figure ce qui peut se produire, pour en envisager ensuite les conséquences. Cet examen verse régulièrement dans le ton de la dramatisation en produisant un sentiment d'irrémédiable.

L'association fréquente de si avec l'adverbe jamais est l'un des outils de cette dramatisation. Placé dans la principale, l'adverbe souligne le caractère irréversible de l'enchaînement : «Si elle prend la chose de travers, je me perds à jamais» (La Chartreuse de Parme, p. I I 7). Rattaché à si dans la subordonnée, il contribue à dramatiser la situation décrite :

Si jamais Fabrice s'échappe [...], l'incommensurable lâcheté du marquis del Dongo trouvera qu'il a du péché à envoyer du pain à un homme poursuivi par un prince légitime [...]. (La Chartreuse de Parme, p. 283-284)

L'emploi fréquent du présent dans la principale contribue également à accrô̂tre le caractère inéluctable de la suite envisagée et rend perceptibles l'accélération des battements cardiaques du personnage et son ton angoissé :

Mais si je ne commets cette imprudence, demain, je ne puis la voir. (La Chartreuse de Parme, p. 320)

Plutôt que de recourir au futur que l'adverbe demain appellerait, Fabrice préfère un présent de l'indicatif faisant ressortir le lien de cause à conséquence réunissant les deux propositions. Ce lien obéit selon le personnage à une logique rigoureuse qui peut s'énoncer, comme la logique mathématique, au présent.

Les articulations logiques des monologues cristallisent le bouleversement de l'individu et son effort pour se reprendre et raisonner malgré tout. Écoutons Mosca, dévoré par la jalousie, essayer de retrouver la raison: 
Il faut se calmer; si j'ai des manières rudes, la duchesse est capable, par simple pique de vanité, de le suivre à Belgirate; et là, ou pendant le voyage, le hasard peut amener un mot qui donnera un nom à ce qu'ils sentent l'un pour l'autre; et après, en un instant, toutes les conséquences.

La solitude rendra ce mot décisif, et d'ailleurs, une fois la duchesse loin de moi, que devenir? et si, après beaucoup de difficultés surmontées du côté du prince, je vais montrer ma figure vieille et soucieuse à Belgirate, quel rôle jouerai-je au milieu de ces gens fous de bonheur? (La Chartreuse de Parme, p. I 57. Nous soulignons)

Recourant tour à tour à la conjonction de subordination si et à la conjonction de coordination $e t$, Mosca élabore le récit d'un enchaînement fatal : son dépit aura pour corollaire certain le départ de la duchesse, qui provoquera l'aveu mutuel de l'amour entre la tante et le neveu et l'isolement irrémédiable du comte. Le ton tragique de ce monologue résulte de cette mise en relation, où les propositions comme les événements se déduisent les uns des autres dans un mouvement irréversible.

Le ton est le produit de cette mise en tension des événements. Il procède des rapports que l'esprit postule entre les événements, rapports qui semblent obéir à une logique objective et irrévocable. Mosca n'a nullement conscience d'échafauder un récit à partir d'une série d'hypothèses. Le ton tragique tient au contraire à sa certitude d'être face à la logique implacable d'événements emboîtés les uns dans les autres.

Peut-être tient-on là un des aspects du «ton du Code civil ${ }^{15}$ » que Stendhal donnait à Balzac comme son modèle d'écriture, avant de confesser que ses maladresses stylistiques tenaient à son «amour exagéré pour la logique» (Correspondance, t. III, p. 399). En effet, le Code civil, notait Michel Pêcheux, est le modèle de l'écriture à enchâssement : la détermination et l'explication s'encastrent «comme les pièces d'un mécanisme indestructible fonctionnant dans l'éternité de l'évidence logico-juridique ${ }^{16} \gg$. Tout se tient : tel fait appelle inévitablement telle procédure et tel type de sanctions ${ }^{17}$.

La tension est celle du désir, qui s'inscrit dans les mots de liaison. Il est singulier de voir comment le désir avance masqué dans les articulations d'un raisonnement qui se targue d'être le plus objectif possible. Ainsi, toute une

I s. «En composant la Chart[reuse], pour prendre le ton, je lisais de temps en temps quelques pages du Code civil» (Correspondance, t. III, p. 399. Lettre à Balzac des 17-28 octobre I 840).

i6. Fr. Gadet, M. Pêcheux, La Langue introwvable, François Maspero, I98 I, p. I47.

17. Une précision s'impose : M. Pêcheux parle du Code civil dans sa version actuelle, plus abstraite. Le Code du début du XIX ${ }^{\mathrm{e}}$ siècle était plus prescriptif, plus concret. Cependant, un rapide examen du Code civil de I82 I (Code civil annoté, Paris, Imprimerie d'Hautel, I 82 I) confirme la fréquence des phrases à subordonnées et à enchâssement. 
partie du long monologue de Gina après l'arrestation de Fabrice démontre «mathématiquement» qu'il faut s'éloigner du comte, sans souffler mot de la raison véritable : son envie irrépressible de l'écarter de son intimité avec Fabrice et d'être en tête-à-tête avec l'homme qu'elle aime.

La prudence méticuleuse du comte gênerait tous mes projets, et d'ailleurs il ne faut point l'entraîner dans ma perte [...] Car pourquoi la vanité de ce tyran ne me jetterait-elle pas en prison? J'aurai conspiré, quoi de plus facile à prouver? Si c'était à la citadelle qu'il m'envoyât et que je pusse à force d'or parler à Fabrice, ne fût-ce qu'un instant, avec quel courage nous marcherions ensemble à la mort! (La Chartreuse de Parme, p. 284. Nous soulignons)

Après avoir suggéré que le caractère de Mosca perturberait ses plans, la duchesse s'engage dans un autre ordre de réflexions et considère l'emprisonnement qui la menace - la locution adverbiale d'ailleurs souligne ce déplacement. Car introduit un long développement sur les risques de son incarcération, développement qui n'est pas sans jouissance : Gina rêve d'être emprisonnée avec Fabrice, enfin seuls et unis jusqu'à la mort. Une oreille attentive sent le désir affleurer dès l'énoncé du car, derrière la façade de raisonnement objectif. Gina songe longuement à ce possible emprisonnement, puis elle revient à la question de ses rapports avec le comte et tranche :

Oui, il faut me brouiller très ostensiblement avec le comte, car je ne veux pas l'entraîner dans ma perte, ce serait une infamie; le pauvre homme m'a aimée avec tant de candeur! (La Chartreuse de Parme, p. 284. Nous soulignons)

Les menaces d'incarcération deviennent maintenant la raison rendant la rupture nécessaire. Par un curieux phénomène de déplacement, carn'introduit plus la probabilité d'incarcération de la duchesse mais la nécessité de se brouiller avec Mosca : la duchesse, magnanime, se refuse à l'entraîner dans sa perte. Car fait plus que «sauter une idée intermédiaire ${ }^{18}$ » (J. Prévost, op. cit., p. 5 I6) : par sa ductilité, il donne au monologue le ton de la mauvaise foi. En effet, la duchesse opère grâce à lui un habile renversement de l'ordre des désirs et des nécessités, faisant passer son désir d'éloigner Mosca pour une nécessité vitale indépendante d'elle.

Le ton de la dramatisation, le ton du désir ou de la peur procèdent donc de l'articulation des propositions, articulation censée traduire le lien inéluctable entre les événements. Est-ce à dire, à l'inverse, que le ton détaché résulte d'une écriture de la dé-liaison, du délié? Nous allons voir que le phénomène est plus complexe, que le ton détaché des monologues stendhaliens tient tout autant aux articulations du discours.

I 8. «Stendhal fait de car un emploi assez fréquent et très personnel. Car sert à sauter une idée intermédiaire. "Cette habitude (de ne pas se laisser payer de vaines paroles) est un crime ; car tout bon raisonnement offense"» (Le Rouge et le Noir, t. I, 27). 


\section{Détachement ou décrochement? Ton et intervalle}

À l'autre bout du spectre, l'étude des monologues dont le ton se veut détendu, détaché, pose des problèmes encore plus épineux. En effet, contre toute attente, ce détachement du ton ne se marque nullement par un détachement des segments phrastiques au sein des monologues. Le ton détaché procède d'un certain mode d'articulation logico-syntaxique. De plus, pour brouiller encore davantage les choses, le ton badin, léger ou cynique est bien souvent affecté dans les discours intérieurs. Il laisse d'ailleurs généralement sourdre, malgré lui, l'angoisse à laquelle le personnage cherche en vain à imposer silence.

L'interjection eh bien participe au premier plan à cette impression de ton détaché. Eh bien est utilisé par le locuteur pour effectuer une thématisation des phrases précédentes servant de support à l'énoncé suivant. Dans ce geste de récapitulation et de conclusion, eh bien indique le ton lucide de l'homme qui se rend à l'évidence. Après avoir récapitulé la situation, le personnage en tire froidement les conséquences. Il considère les choses avec détachement et s'incline devant la nécessité des faits. Le ton apaisé de Julien en prison se perçoit dans l'emploi fréquent de cette interjection. On se souvient par exemple de la réplique impromptue qui met fin aux rêves ambitieux de M. Sorel de la Vernaye, qui se voit ambassadeur...

Mais aussi, quelle perspective!... Colonel de hussards, si nous avions la guerre; secrétaire de légation pendant la paix; ensuite ambassadeur... [...] Homme de mérite et jouissant de la plus grande existence à Vienne ou à Londres...

- Pas précisément, monsieur, guillotiné dans trois jours.

Julien rit de bon cœur de cette saillie de son esprit. En vérité, l'homme a deux êtres en lui, pensa-t-il. Qui diable songeait à cette réflexion maligne?

Eh bien, oui, mon ami, guillotiné dans trois jours, répondit-il à l’interrupteur. (Le Rouge et le Noir, p. 784-785. Nous soulignons)

Mais ce détachement est plus souvent feint que réel. Observons ainsi Mathilde, avec laquelle Julien vient de rompre toute relation, soupirer sur un ton grandiloquent :

Eh bien, tout est fini, se dit-elle avec un calme apparent; il me reste un grand exemple; cette erreur est affreuse, humiliante! elle me vaudra la sagesse pour tout le reste de la vie. (Le Rouge et le Noir, p. 68I)

C'est «avec un calme apparent» fort suspect que Mlle de la Mole prend acte du départ de son amant et en tire des leçons pour le reste de son existence... Le marquis de la Mole évoque de même son attachement pour Julien sur un ton léger qui ne convainc guère :

On s'attache bien à un bel épagneul, se disait le marquis, pourquoi ai-je tant de honte de m'attacher à ce petit abbé? il est original. Je le traite comme un fils; eb bien! 
où est l'inconvénient? Cette fantaisie, si elle dure, me coûtera un diamant de cinq cents louis dans mon testament (Le Rouge et le Noir, p. 597. Nous soulignons)

Le ton insouciant du marquis est largement joué. La comparaison légère avec l'épagneul, la question qui se veut oratoire ("où est l'inconvénient?»), la dénomination de «fantaisie» ne trompent personne : M. de la Mole est profondément troublé par son amitié pour son secrétaire.

En dépit de l'évidence affichée, le lien établi par eb bien entre les énoncés qui précèdent et l'ultime énoncé qu'il introduit est souvent précaire. Le ton complexe de cette interjection tient à l'élasticité de ce lien. Comme l'a noté Young-ai Hwang, eh bien dans le discours parlé marque souvent une «rupture de consensualité» et le passage «à un développement personnel ${ }^{19}$ ». Eh bien est le signe d'affirmation d'une personnalité qui affiche hautement une conclusion qu'elle sait inattendue. Les eh bien des monologues de Mathilde sont souvent déclamés sur un ton tout solennel :

Au milieu des transports les plus vifs, quand elle serrait contre son cœur la tête de Julien : Quoi! se disait-elle avec horreur, cette tête charmante serait destinée à tomber! Eh bien! ajoutait-elle enflammée d'un héroïsme qui n'était pas sans bonheur, mes lèvres, qui se pressent contre ces jolis cheveux, seront glacées moins de vingt-quatre heures après (Le Rouge et le Noir, p. 772. Nous soulignons)

Loin de repousser l'idée de la mort prochaine, Mathilde se complaît dans un scénario macabre qu'elle se raconte intérieurement. La solennité de ton de eh bien se veut à l'image de la fermeté de caractère d'une jeune fille peu commune qui sait regarder l'avenir en face et en tirer courageusement les conséquences : le suicide l'attend, comme seule fin envisageable.

Mais à d'autres moments, les renversements, les nœuds que recouvre cette locution sont moins assumés. La complexité du ton découle de ce flottement que perçoit confusément le lecteur. Penchons-nous par exemple sur le monologue où Mathilde répond aux plaisanteries méprisantes que les jeunes nobles, MM. de Luz, Croisenois, adressent à Julien et à son «air prêtre» :

Malgré tous les désavantages de son éternel habit noir et de cette physionomie de prêtre, qu'il lui faut bien avoir, le pauvre garçon, sous peine de mourir de faim, son mérite leur fait peur, rien de plus clair. Ét cette physionomie de prêtre, il ne l'a plus, dès que nous sommes quelques instants seuls ensemble. Et quand ces messieurs disent un mot qu'ils croient fin et imprévu, leur premier regard n'est-il pas pour Julien? je l'ai fort bien remarqué. Et pourtant ils savent bien que jamais il ne leur parle, à moins d'être interrogé. [...] Eh bien, mon père homme supérieur, et qui portera loin la fortune de notre maison, respecte Julien. (Le Rouge et le Noir, p. 632. Nous soulignons)

I9. Young-ai Hwang, "Eh bien, alors, enfin et disons, en français parlé contemporain», L'Information grammaticale, $\mathrm{n}^{\circ} 57$, mars i 993, p. 46. 
Mathilde, mal à l'aise, éprouve le besoin de contrer ces railleries, sans donner pour autant à son discours intérieur un tour argumentatif trop marqué : le détachement dont elle se targue vis-à-vis des jugements étriqués de son milieu lui interdit toute argumentation ouverte.

Les Et en tête de phrase revêtent dans cet extrait l'assurance de ton de la personne lucide qui sait considérer les situations et en faire ressortir les paradoxes. Mathilde prétend ne faire que constater les faits sur un ton distant: ces jeunes nobles sont tout simplement tiraillés par l'envie et soumis au regard d'approbation d'un secrétaire n'ayant pas droit à la parole.

Pourtant, le sentiment refoulé fait retour à la fin du monologue, lorsque Mathilde oppose à la critique des jeunes nobles l'avis de son père : «Eh bien, mon père homme supérieur, et qui portera loin la fortune de notre maison, respecte Julien.» (Le Rouge et le Noir, p. 632. Nous soulignons) Eh bien marque une disjonction entre la première partie du monologue qui affiche l'indifférence de Mathilde face aux verdicts de son milieu et la dernière phrase qui l'oblige à se tourner vers le marquis de la Mole. Mathilde a besoin de la caution familiale et sociale, dont elle n'est point détachée. D'ailleurs, l'autorité morale du marquis repose sur sa capacité à «porte[r] loin la fortune de notre maison». Toute l'angoisse de Mathilde qui trahit sa lignée en jetant son dévolu sur un homme pauvre rejaillit dans cette dernière phrase.

Eh bien rend cette angoisse sensible, derrière le ton apparemment léger et détaché. Au vu de celle-ci, tous les et qui précèdent peuvent être traduits par des mais: la jeune femme négocie silencieusement avec les normes et les impératifs de son milieu, normes qui l'habitent et alimentent sa honte du «pauvre garçon» (Le Rouge et le Noir, p. 632). L'articulation est verticale, entre ces normes intériorisées et le discours que l'héroïne se tient à elle-même. La coordination se fait entre des niveaux hétérogènes, entre le non-dit et le discours explicite. En cela, les articulations de la phrase montrent tous les nœuds, tous les sentiments du sujet, y compris ceux qu'il voudrait taire.

Le rythme passe par la liaison des idées, mais cette liaison enjambe régulièrement des failles intérieures qu'elle rend sensibles, plus qu'elle ne les colmate. Le rythme engage le sujet tout entier et laisse percevoir les doutes et les angoisses en même temps que la volonté de les étouffer. Paradoxalement, les mots de liaison deviennent alors les indices de disjonctions. Ce pourra être le brutal décrochage entre l'amour que Lucien croyait partager avec Bathilde et la déception provoquée par l'apparition de Mlle Bérard, qui met fin chez lui à tout désir :

Il était à cent lieues du souvenir de Mme de Chasteller, il y revint :

... Et non seulement je croyais l'aimer, mais je croyais voir clairement qu'elle a pour moi un commencement d'affection. 
En ce moment il eût pensé à tout avec plus de plaisir qu'à Mme de Chasteller. C'était la première fois depuis trois mois qu'il se trouvait en présence de cette étrange sensation. (Lucien Leuwen, p. 267-268. Nous soulignons) ${ }^{20}$

Le Et initial ne relance rien, en l'occurrence. Il est le vecteur du ton d'amertume de Lucien, de son vertige face au gouffre séparant ses sentiments passés de son état présent. «Il y a, manifestement, chez Stendhal comme dans la langue, un polymorphisme pervers du et, tour à tour (ou à la fois) additif, oppositif, consécutif ou "vide".» note finement Georges Kliebenstein", avant d'ajouter : "Le et stendhalien, en régime microstructural [...] semble voué, en somme, à relier ce qui ne devrait pas l'être.» De fait, le Et en tête de phrase, dans les monologues, signale fréquemment un fossé entre deux éléments qu'il s'écartèle à rassembler... ou qu'il enjambe à pieds joints, comme Mme Grandet s'empresse de le faire :

La démarche de Mme Grandet était combinée avec son mari, mais elle s’abstint de rendre compte de la réponse avec la dernière exactitude.

Elle entrevoyait bien qu'il n'eût pas été décidément impossible de l'amener à une façon raisonnable, et philosophique, et politique, de voir les choses, mais c'est toujours une discussion terrible pour une femme qui se respecte $e t$, se dit-elle, il vaut bien mieux la sauter à pieds joints ${ }^{22}$.

Et est à l'image du geste par lequel Mme Grandet écarte toute discussion avec son mari et fait taire toute objection personnelle. Il laisse sentir la faille, les réticences intérieures et le mouvement de refoulement. Le ton résulte précisément de la césure que le lecteur perçoit entre le discours intérieur et le non-dit. Si le ton, en musique, est défini par l'intervalle entre deux notes consécutives de la gamme, le ton du monologue se perçoit dans le fossé qui se creuse entre le discours explicite à soi-même et les objections passées sous silence, que le lecteur entend malgré tout, dans le grand écart qu'effectuent les mots de liaison.

Dans son étude du tempo de La Chartreuse de Parme, Éric Bordas constatait que la juxtaposition et la parataxe allaient de pair dans la narration stendhalienne avec une liaison souterraine, silencieuse, qui assurait la cohésion d'ensemble du récit, comme le jeu legato, qui lie le plus possible les notes entre elles $^{23}$. Le ton des monologues, à notre sens, découle du mouvement inverse.

20. Mme de Chasteller a pensé qu'il était raisonnable qu'elle s'entoure, lors des visites de Lucien, d'une dame de compagnie, Mlle Bérard, dont la présence rend impossible toute expression de leur amour.

21. G. Kliebenstein, «Stendhal et la rhétorique», dans É. Bordas et Ph. Berthier éd., Stendhal et le style, Presses Sorbonne Nouvelle, 2005.

22. Lucien Leunen, p. 644. Nous soulignons. La «démarche» consiste, on le rappelle, à donner son corps à Lucien contre l'obtention d'un portefeuille de ministre pour M. Grandet.

23. É. Bordas, «Stendhal bel cantiste. Tempo et legato dans La Chartreuse de Parme», dans D. Sangsue éd., La Chartreuse de Parme. Chant et tombeau, Recherches \& Travaux, hors série n I3, Grenoble, 1997, p. 71. 
L'explicitation des articulations logiques, leur soulignement, s'accompagnent à maintes reprises d'une série de décrochages entre l'ordre du non-dit et le discours à soi-même. Le personnage s'emploie alors à enjamber cette faille intérieure et à enchaîner malgré tout. Les désirs sont mis au pas, ramenés à une forme lisse. Le ton est «une digue ${ }^{24} »$, écrivait Stendhal à propos du «bon ton» que la société se doit d'adopter, une police du langage qui réprime les mouvements passionnels. À leur manière, les mots de liaison serviraient de balises dans les monologues pour canaliser désirs et pulsions, leur légère distorsion en témoigne.

24. Correspondance, t. I, p. 570. Lettre à Pauline Beyle du 23 mai i 8 I o. 